\title{
Distances and directions are computed separately by honeybees in landmark-based search
}

\author{
KEN CHENG \\ Macquarie University, Sydney, Australia
}

\begin{abstract}
Honeybees were trained with two landmarks at some angle (e.g., $120^{\circ}$ ) apart from the target. On crucial unrewarded tests, only a single landmark was present. If distances and directions to landmarks are computed separately (independent averaging), the search distance to the landmark should equal the landmark-target distance found in training. If entire vectors are averaged, the search distance should be much shorter. Three experiments with short target-landmark distances showed results in between the predictions of the two hypotheses. A fourth experiment used longer target-landmark distances and isolated double peaks on single-landmark tests: one predicted by the independent averaging hypothesis, and one very close to the landmark. The near peak is interpreted as arising from approach and exploration of a landmark in a new location, and not from searching.
\end{abstract}

Finding places again is crucial in the lives of many animals. Survival and the passing on of genes often depend on finding a nest, a home, or a foraging site again. Two common methods for finding a place are path integration and landmark-based spatial memory. The two are often used in combination (e.g., Etienne, Joris-Lambert, DahnHurni, \& Reverdin, 1995; Etienne, Joris-Lambert, Maurer, Reverdin, \& Sitbon, 1995; Etienne, Teroni, Hurni, \& Portenier, 1990).

Path integration is a method for getting back to a starting point, typically home. The animal keeps track of the straight-line distance and direction from the starting point to its current location. When the time comes, this vector information is used to chart the way home (for reviews, see Etienne, Berlie, Georgakopoulos, \& Maurer, 1998; Gallistel, 1990, chap. 4). This method of placefinding has been found in insects (e.g., Müller \& Wehner, 1988; Wehner, Michel, \& Antonsen, 1996; Wehner \& Srinivasan, 1981; Wehner \& Wehner, 1990) and rodents (e.g., Etienne, Joris-Lambert, Dahn-Hurni, \& Reverdin, 1995; Etienne, Joris-Lambert, Maurer, et al., 1995; Etienne et al., 1990).

In landmark-based spatial memory, the topic of this paper, the navigating animal codes the target location by some of its spatial relations to surrounding landmarks (for reviews, see Gallistel, 1990, chap. 5; Healy, 1998). Finding the target means moving so as to restore the spatial relations between landmarks and target (Cheng, 1995). Distant landmarks may be used to define which direction is which (e.g., honeybees, Dyer \& Gould, 1983). Land-

This research was supported by grants from Macquarie University and the Australian Research Council. I thank Thomas Collett and an anonymous reviewer for suggesting the crucial Experiment 4, Charles Blaha for comments on an earlier draft, and Dagong Zhang for conducting experiments and analyzing data. Correspondence should be addressed to K. Cheng, Department of Psychology, Macquarie University, Sydney NSW 2109, Australia (e-mail: kcheng@axon.bhs.mq.edu.au). marks close to the target may be used to pinpoint the target location. The latter strategy is ubiquitous, being found across the animal kingdom-in ants (Wehner \& Räber, 1979), digger wasps (Tinbergen, 1972), wasps (Collett \& Rees, 1997), honeybees (Cartwright \& Collett, 1982, 1983; Cheng, Collett, Pickhard, \& Wehner, 1987; Cheng, Collett, \& Wehner, 1986; Collett \& Baron, 1994; Collett \& Kelber, 1988; Collett \& Rees, 1997; Gould, 1987; Huber, Couvillon, \& Bitterman, 1994; von Frisch, 1953; for reviews, see Collett, 1992; Collett \& Zeil, 1998), cephalopods (Mather, 1991), Clark's nutcrackers (GouldBeierle \& Kamil, 1996; Kamil \& Jones, 1997; Vander Wall, 1982), black-capped chickadees (Brodbeck, 1994; Cheng \& Sherry, 1992), European jays (Bennett, 1993), chicks (Vallortigara, Zanforlin, \& Pasti, 1990), pigeons (Cheng, 1988, 1989, 1990, 1994; Cheng \& Sherry, 1992; Cheng \& Spetch, 1995; Spetch, 1995; Spetch, Cheng, \& MacDonald, 1996; Spetch et al., 1997; Spetch, Cheng, \& Mondloch, 1992; Spetch \& Edwards, 1988; Spetch \& Mondloch, 1993; Spetch \& Wilkie, 1994; see Cheng, 1995, for review), mice (Alyan \& Jander, 1994), hamsters (Etienne, Joris-Lambert, Dahn-Hurni, \& Reverdin, 1995; Etienne, Joris-Lambert, Maurer, et al., 1995; Etienne et al., 1990), gerbils (Collett, Cartwright, \& Smith, 1986), and rats (e.g., Biegler \& Morris, 1993, 1996; Cheng, 1986; Margules \& Gallistel, 1988; Suzuki, Augerinos, \& Black, 1980).

In honeybees, the subject of this paper, research has identified landmark-based spatial memory to be used in the final stage of place finding (Collett \& Rees, 1997). Honeybees are thought to get to the area of the target (in which landmarks for precise localization can be seen) by following a route or vector (Wehner \& Menzel, 1990). Although Gould (1986) found evidence for map-based navigation in honeybees, attempts at replication with more rigorous controls have failed (Dyer, 1991; Wehner, Bleuler, Nievergelt, \& Shah, 1990; Wehner \& Menzel, 1990; but see Menzel, Geiger, Jeorges, Müller, \& Chittka, 
1998). Working with both bees and wasps, Collett and Rees (1997) identified three steps in getting to the target once the insects were in the region of it. First, the insect heads toward identified landmarks (beaconing), typically from about the same direction. Second, when a landmark appears at a particular site, it takes a particular trajectory that leads it to the vicinity of the target (sensorimotor trajectory). Third, it flies facing one direction and attempts to place the surrounding landmarks at the right locations on the eyes (landmark matching, Collett \& Baron, 1994; Collett \& Rees, 1997).

The sensorimotor trajectory has been identified only recently and requires perhaps more confirmatory evidence. At the moment, it is based on some observations in one paper (Collett \& Rees, 1997). The landmarkmatching process has been well studied. Evidence suggests that the honeybees encode and use both the compass direction and the distance from the target to landmarks (Cartwright \& Collett, 1982, 1983). The problem of defining compass direction is reduced to coding by retinal position because the bee flies facing one direction (Collett \& Baron, 1994). Honeybees can tell the distance to an object by the retinal size projected by an object (Cartwright \& Collett, 1982, 1983) and motion parallax (Lehrer, Srinivasan, Zhang, \& Horridge, 1988; Srinivasan, Lehrer, Zhang, \& Horridge, 1989). Early models of landmark matching (Cartwright \& Collett, 1982, 1983) identified retinal size as the key determinant of distance, but later research identified a role for motion parallax as well (Cheng et al., 1987; Lehrer \& Collett, 1994). Landmarks are also thought to be identified by color (Cheng et al., 1986; Gould, 1987). Unpublished results gathered by Cheng et al. in 1986 also revealed no evidence that honeybees could identify 3-D landmarks by shape alone.

Cartwright and Collett's $(1982,1983)$ models also posited two other characteristics in searching. One is that the honeybee attempted to match both the direction and the size of individual landmarks and the interlandmark angles. So far, research in progress in my lab and research by Collett and Rees (1997) have yielded evidence for matching individual landmarks. Whether and how interlandmark angles are used remains unclear.

A second claim of Cartwright and Collett's $(1982,1983)$ model is that distances and directions are computed separately and independently. The experiments reported here test this claim by adopting methods used on pigeons (Cheng, 1994) and on trajectory learning in honeybees (Collett \& Baron, 1995; Collett, Baron, \& Sellen, 1996). The basic experimental logic is illustrated in Figure 1. The subject is presented with a directional conflict. Two cues point to different directions, but at the same distance, from a landmark. The crucial tests in the experiments reported had only one landmark. The bee can be assumed to be aiming to get to a target place according to the landmark. Two dictates stem from the landmark. They are represented as landmark-target vectors (arrows in Figure 1), emanating from the landmark, with the endpoints representing the target location according to two

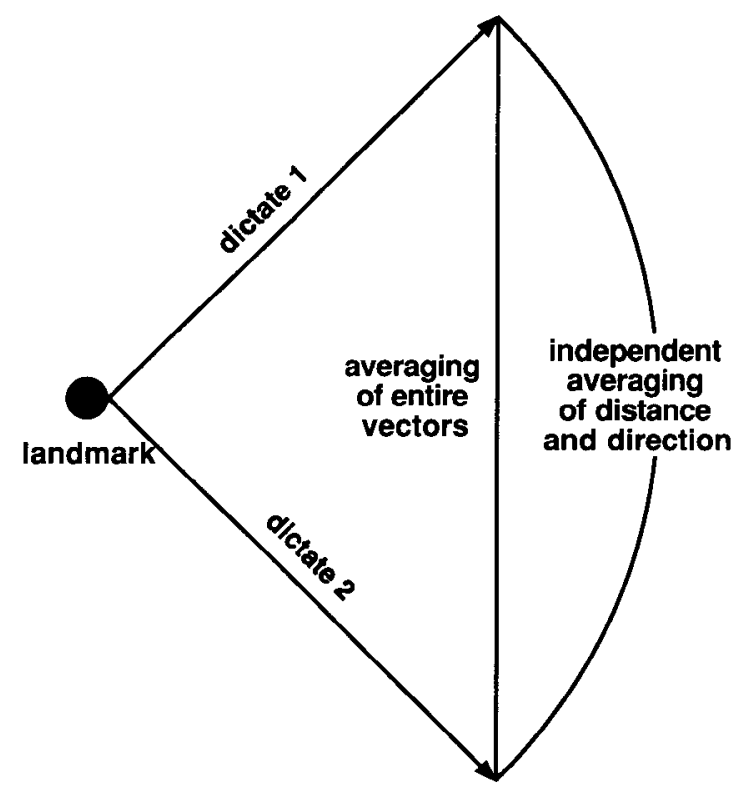

Figure 1. Two ways of averaging two vectors from a landmark. In averaging entire vectors, the average must lie on the line segment connecting the endpoints of the vector. In averaging distances and directions separately, the average must lie on the arc connecting the endpoints of the vectors.

different cues. The subject is presumed to average the dictates of the two cues and to search at an intermediate location. The question is whether the subject averages entire vectors, distance and direction taken together (vector averaging), or averages the distance and directional components separately and independently (independent averaging). If the entire vectors are averaged, the weighted average must lie on the straight line segment connecting the endpoints of the two vectors; this follows from vector geometry, and a proof is given in the Appendix. If distances and directions are averaged separately, the weighted average must lie on the arc connecting the endpoints of the two vectors, preserving the radial distance to the landmark (the vector length).

Such experiments have been conducted on landmarkbased search in pigeons (Cheng, 1994). For example, the target was $13.5 \mathrm{~cm}$ east of a bottle (landmark). A black cardboard strip on the bottle faced the target. On manipulated tests, the bottle and the strip along with it was turned by $90^{\circ}$, thus creating a $90^{\circ}$ directional conflict between the dictates of the strip and the rest of the landmark array. The experiments clearly supported the independent averaging hypothesis.

Collett et al. (1996), on the other hand, tested honeybees on averaging trajectories and found clear evidence for vector averaging. In these tasks, honeybees entering a test chamber had to take a particular vector to get through to the reward; that is, they had to travel a particular distance in a particular direction in order to drop through a hole on the perspex bottom to the next chamber (and eventually to the reward). Each vector trajec- 
tory was associated with a stimulus pattern on the back wall of the chamber. The stimuli were oriented stripes. For example, stripes oriented oblique right might dictate a vector of $50 \mathrm{~cm}$ at $60^{\circ}$ right, while stripes oriented oblique left might dictate a vector of $50 \mathrm{~cm}$ at $60^{\circ}$ left. The bees had to learn sensorimotor trajectories. To create directional conflict, stripes at intermediate orientations (e.g., vertical stripes) were presented on some tests, and the trajectories of the honeybees were noted. The data clearly supported the vector averaging model. Averaging is a form of adding - adding weighted vectors in this case. In adding egocentric vector trajectories, it makes sense to use vector addition. Separate addition of distances and directions does not in general yield the correct answer. For instance, in adding 5 steps east to 5 steps north, we do not get 10 steps northeast (or 10 steps in any direction). The only correct way to add egocentric trajectories is to add entire vectors, using vector addition.

In the experiments reported here, the same experimental logic was applied to landmark-based search in honeybees. On the basis of results from pigeons (Cheng, 1994) and from the claims of Cartwright and Collett's models $(1982,1983)$, the prediction would be independent averaging. In estimating distance and direction from a landmark, the two components may well be calculated separately. In this modular fashion, different specialized neural and computational units may be devoted to each task. On the other hand, the honeybees are computing over entire vectors in the case of learning trajectories, and sensorimotor trajectories are thought to form a part of landmark-based search (Collett \& Rees, 1997). Both of these points suggest that vector averaging may prevail.

\section{GENERAL METHOD}

\section{Setup}

The honeybees (Apis mellifera) lived in a hive just outside the lab building in which they were tested. The lab moved in the course of research, and Experiment 1 was conducted in the old lab on the south side of the building, whereas Experiments 2-4 were conducted in the new lab on the north side of the building. The two labs differed in terms of entrance direction, the wall colors, and furniture. The test arena, however, was the same table in both labs.

At each site, a feeding station providing sugar water was set up for the bees on a table outside the entrance window of the lab. The sugar water was typically one part sugar to four parts water, but was varied in strength to attract more or fewer bees. The entrance to the lab was a $2-\mathrm{cm}$ square hole cut out of a specially constructed perspex bee-entrance system. A guillotine-style perspex "gate" allowed entrance control for experimenters. Through the hole ran a gangplank, painted with stripes of red nail polish for visibility. To recruit subjects, the plate with sugar water was temporarily placed right next to the entrance until a few bees found the rewardstrength sugar water (one part sugar to one part water) in a translucent bottle cap (2.5-cm diameter) on the outside part of the gangplank. The reward dish was then moved to the inside part of the gangplank. Bees that returned to the lab were individually marked for identification.

The insects' final destination in the lab, after they had walked through the hole on the gangplank, was a table $(120 \times 75 \times 73 \mathrm{~cm}$ high, with the long edge parallel to the wall under the window).
The table was covered with white poster paper with squiggles drawn on it in thick felt-tip pen (black in Experiments 1-3, yellow in Experiment 4). The squiggles helped to stabilize the flights of honeybees over the otherwise featureless surface; they have been used similarly in past studies (Cartwright \& Collett, 1982, 1983; Cheng et al., 1987; Cheng et al., 1986). From the beginning, the setups on the table were the training setups used for the experiment. Landmarks used were cylindrical bottles covered with light-yellow or light-blue paper. Particular setups used are described in each experiment. The entire setup of cap and landmarks changed locations on the table throughout experimentation, so that the experimental landmarks were the only reliable indicators of the exact location of the reward. After the forager consumed the reward to repletion, she flew to the window and was released through a swinging door (10cm square) above the entrance.

The lab rooms each contained plenty of cues indicating direction. These included a stand for the black-and-white video camera above the table, which straddled the two short sides of the table, a window, a door, video equipment, and other furniture. Overhead fluorescent lights lit each room.

\section{Training and Testing}

The insects encountered one or two arrays of landmarks during training. On each trial, only one array was encountered. The training procedure followed the routine of past studies (Cheng et al., 1987; Cheng et al., 1986) and took place during daylight hours, typically between 9:00 and 16:30. On early trials, a subject was moved from the entrance window to the correct place on the table. After a few such trials, the forager was released at a small distance from the goal. Then she was released beyond the edge of the table, the final training condition. Training in general took $1 / 2$ to 1 full work day.

Tests were then mixed with standard training trials. On training trials, a number of bees might be performing simultaneously, but on tests, only a single subject was admitted. Others were either shut out by the gate or temporarily captured in a matchbox. Tests were given about every third trial, with variations arising from inevitable scheduling jams in running 3-5 animals at the same time. This served well in providing some degree of randomness to the number of training trials between tests. On a test, the goal (cap) was absent. The landmark setup might be altered. Each experiment, however, included control tests in which the landmark setup was identical to an array encountered during training trials. When a strip of cardboard was laid on the table during training (in Experiments 2-4), it was replaced with a replica for all tests so that odors and other cues left on the strip were removed for tests. The location of the test array varied from test to test, but was central on the table under the video camera. The bee's search was videotaped for $60 \mathrm{sec}$ on VHS tape from the time she entered one of the marked regions, after which the landmarks were restored to the training setup and the testee was allowed to complete a training trial. We planned to give each subject four tests of each type, but weather and quitting bees sometimes cut things short. Bees were given one round of all test types before another round was initiated. With two test types, we alternated test types. With more than two test types, order in each round was determined by a random number table.

\section{Data Analysis}

In Experiments 1-3, the dependent measure was the amount of time (out of $60 \mathrm{sec}$ ) that the bee spent in each region marked on a template used for testing. The lines on the template were drawn in pencil, too thin to be perceived by the bees, whose spatial resolution is estimated at over $3^{\circ}$ (Gould, 1987). The data were obtained from frame-by-frame analysis of the videotaped tests, much as in the past (e.g., Cheng et al., 1987; Cheng et al., 1986). The number of frames in which a bee was in each marked region was counted. 
The VCR used, a Panasonic AG-MD830, delivered high-quality jitter-free freeze frames or images at slow speed, rendering the counting task straightforward if tedious. Given that the videotape recorded 25 frames/sec, dividing the frame count by 25 gave the duration in seconds. Results are expressed as proportions of total test time. In the final experiment, a semiautomatic movement tracking software was used. This is described in the method section of Experiment 4. Most statistical tests were considered significant at $p<.05$. Statistical tests of the vector and independent averaging hypotheses, however, used the same set of data (on single-landmark tests). Alpha level was set conservatively at .01 for these tests, and the $p$ value is mentioned.

\section{EXPERIMENT 1}

The first experiment sought evidence that bees average the dictates of different elements, without testing for the type of averaging (vector vs. independent). In the training setup, two identical yellow landmarks were $120^{\circ}$ apart from the goal, each $10 \mathrm{~cm}$ away (Figure $2 \mathrm{~A}$ ). On a crucial test, only one landmark was present. In the training setup, the forager should encode two elements. One element points to the target at $60^{\circ}$ west of south from it and at $10-\mathrm{cm}$ distance; the other element points to the target at $60^{\circ}$ east of south from it and at $10-\mathrm{cm}$ distance. In the case of a single-landmark test, both elements in the record would be matched to the one landmark present. Averaging the two dictates gives a direction of (approximately) south from the landmark. This means that the honeybees should search more in the region south of the landmark on single-landmark tests than on control tests if they are averaging the dictates of two landmarks.

\section{Method}

This experiment was conducted in the old lab, into which the bees entered from the south. The setup used in training is shown in Figure 2A. The landmarks were bottles wrapped in light-yellow
A
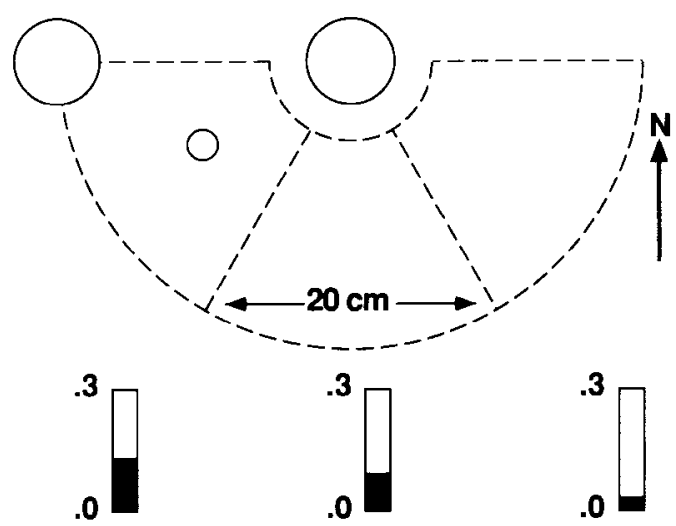

proportion of searching

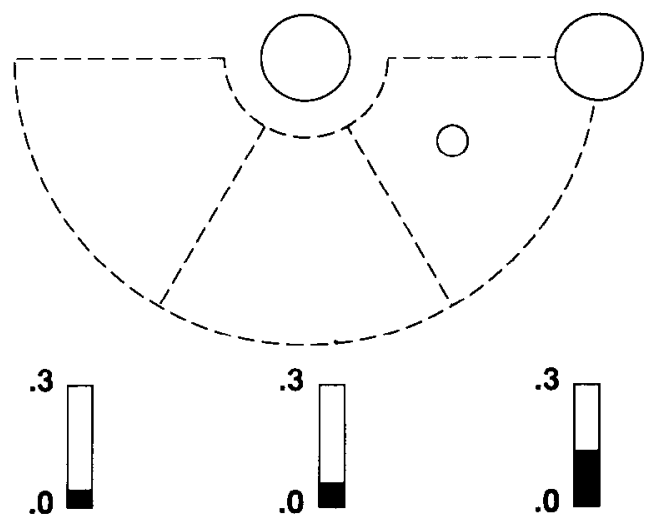

proportion of searching
B

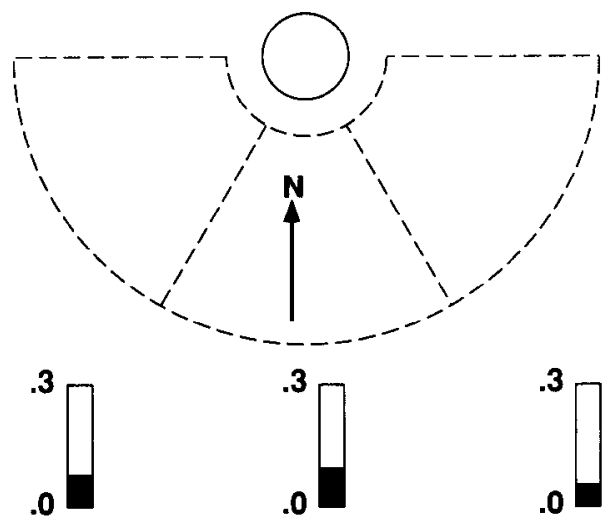

proportion of searching

Figure 2. Setups and results of Experiment 1. The large open circles represent light-yellow cylindrical landmarks. The segments in dotted lines represent a template of areas drawn in pencil in which the presence of the searching bee was counted in a frame-by-frame analysis. The small open circle represents the target (dish of sugar water). Lab entrance is at the bottom of the figure (south). Results are expressed as a proportion of search time on 60 -sec tests. A: Training condition and control tests. B: Single-landmark tests. 
paper ( $7-\mathrm{cm}$ diameter, $15-\mathrm{cm}$ height). Three types of tests were given to 6 animals, tested in two lots of 3 each. Training took 16-20 trials. Two types of control tests were given, differing in where a template was placed: around the west landmark or around the east landmark. The third type of test had a single yellow landmark (Figure $2 \mathrm{~B}$ ). The template was a semicircle placed around the south side of it. The template was divided by pencil into nine $20^{\circ}$ sectors. Semicircles were drawn at 3 - and $20-\mathrm{cm}$ distance from the circumference of the landmark. The nine sectors were collapsed into the three regions shown for data analysis (Figure 2) since the ninefold division was too fine. The averaging hypothesis predicts more searching in the middle sector on single-landmark tests.

\section{Results and Discussion}

The results in Figure 2 show that the honeybees searched more in the middle region on single-landmark tests than on the two control tests. One statistical test is the interaction effect in an analysis of variance (ANOVA) with the three test types and the three locations as factors. The ANOVA indicated that this interaction was significant $[F(4,12)=24.37]$, but revealed no other significant effects. This, however, is not a satisfactory test of the averaging hypothesis. The two control tests have different distributions, arising from the placement of the template. That fact alone would produce a test $\times$ location interaction. A better test is to average the two control distributions to obtain a single distribution to compare against the distribution from single-landmark tests. If the bees did not average, but treated the single landmark sometimes as the east landmark and sometimes as the west landmark, their search distribution on single-landmark tests should resemble the average of the two control distributions (approximately equal proportions of search time in each region). If they averaged the dictates of the two landmarks, however, they would search more in the middle and again produce a test $\times$ location interaction. The two control distributions were thus averaged, and an ANOVA was conducted with two levels of tests (controls combined vs. single landmark) and three locations. The test $\times$ location interaction again came out significant $[F(2,12)=7.95]$, with no main effects found. The averaging hypothesis was thus supported. Searching was too scattered to measure the data on radial distance of search, necessary for testing the vector and independent averaging hypotheses. But this experiment showed that bees do average the dictates of two different landmarks and set the stage for the next three experiments to test which kind of averaging the honeybees used.

\section{EXPERIMENTS 2A AND 2B}

In Experiments 2-4, honeybees were tested in the new lab, which they entered from the north. In Experiment 2, the honeybees were trained with an array of two identical landmarks (Figures 3 and 4). The goal lay between the landmarks on a strip of yellow cardboard. The yellow strip served two purposes: (1) to allow the measurement of search distance from the landmark and (2) to guide the bees in their searching so that the search would be less scattered. In acquiring the task, the bees learned to search primarily on the yellow strip. The measurement of where they searched most along the strip was used to test the independent averaging and vector averaging hypotheses. In two different versions, the perpendicular distance of the goal to the line joining the midpoints of the two landmarks differed. Bees were tested occasionally with either a control test, which consisted of the training setup, or a single-landmark test, in which only one landmark was placed on the yellow strip.

\section{Method}

Eight bees participated in two lots of 4 in Experiment $2 \mathrm{~A}$, and 10 bees participated in two lots of 5 in Experiment 2B. The training setups are shown in Figures 3 and 4 . The landmarks were bottles (7-cm diameter, $15-\mathrm{cm}$ height) wrapped in light-blue paper, $17 \mathrm{~cm}$ apart nearest edge to edge. The strip in between $(7 \mathrm{~cm}$ wide) was light yellow, divided by pencil marks into $5 \times 7 \mathrm{~cm}$ blocks. It ran the width of the table from north to south. The south edge of the landmarks (the back edge from the viewpoint of the entrance) lined up with one of the pencil marks. The position of the target differed in two versions of the experiment. In Experiment $2 \mathrm{~A}$, it was $3 \mathrm{~cm}$ from the tangent connecting the front edges of the bottles. In Experiment $2 \mathrm{~B}$, the equivalent distance was $8 \mathrm{~cm}$. The strip was moved from trial to trial along the length of the table, and the bottles were moved with the strip to maintain the usual spatial re-

A
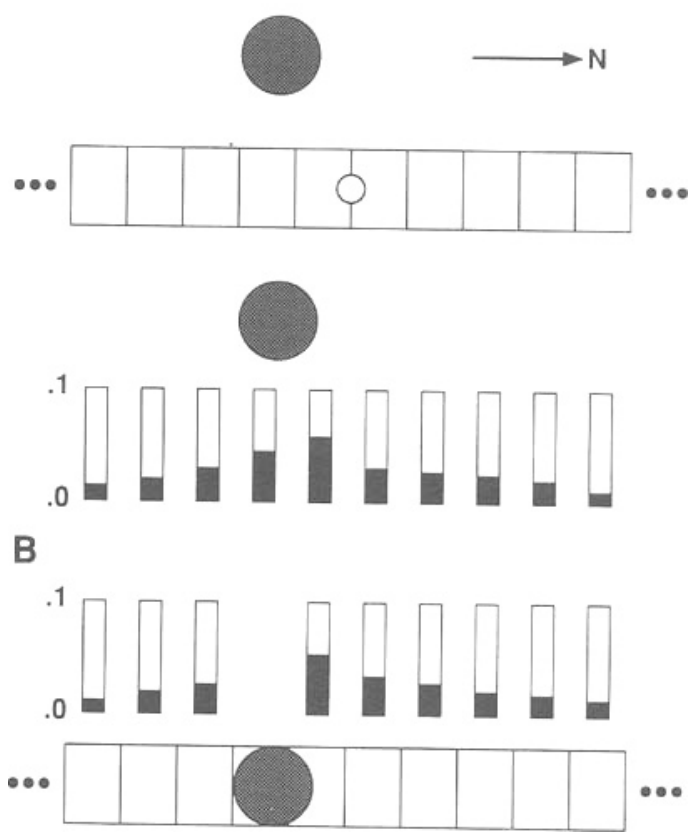

Figure 3. Setups and results of Experiment 2A. The filled circles represent light-blue cylindrical landmarks. The strip was light yellow, with pencil lines drawn $5 \mathrm{~cm}$ apart. Dots indicate that the strip continued the width of the table. The small open circle represents the target (dish of sugar water). Lab entrance is at the right of the figure (N). Results are expressed as a proportion of search time on 60-sec tests. A: Training condition and control tests. B: Single-landmark tests. 
A

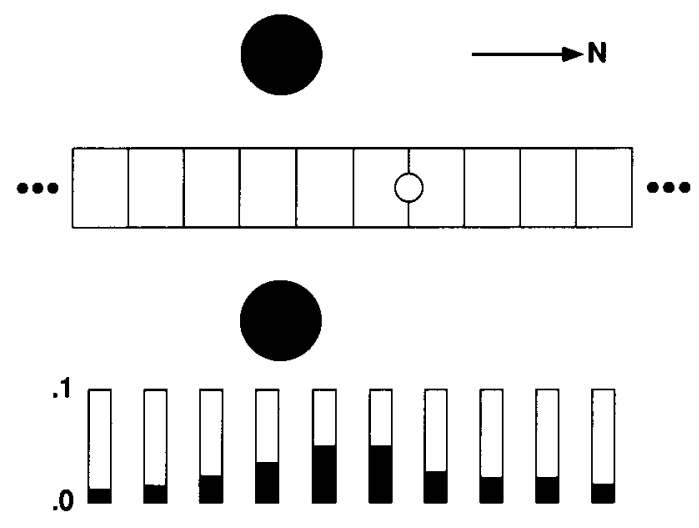

B

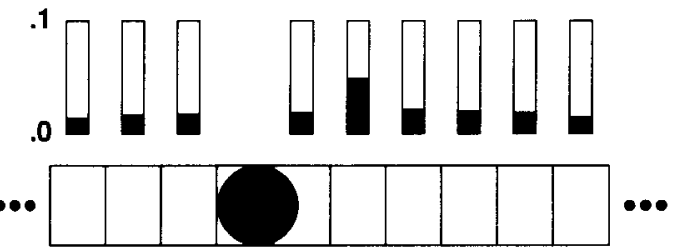

Figure 4. Setups and results of Experiment 2B. Same conventions as Figure 3.

lations. The bottles were also moved along the length of the strip from trial to trial. Training took 16 to 20 trials.

Control tests and single-landmark tests were given. On control tests, the bees faced the training setup without the reward. On single-landmark tests, one bottle was placed on the yellow strip. The south edge of the bottle was on a line on the grid, as it would have been had a bottle in the training setup been shifted along the long dimension of the table.

\section{Results and Discussion}

The distributions of searching on control and singlelandmark tests are shown in Figures 3 and 4 for Experiments $2 \mathrm{~A}$ and $2 \mathrm{~B}$, respectively. Bins beyond those shown were not counted since they were mostly outside the range of the camera. Inspection of the data suggests that the peak of searching was displaced further away from the landmark on single-landmark tests than on control tests. The search distance on single-landmark tests, however, fell short of the radial distance to each landmark on control tests. Because distance on control tests was measured perpendicularly to the line joining the two landmarks, the vector averaging hypothesis predicts the same search distance on the control and single-landmark tests. The independent averaging hypothesis predicts a search distance on single-landmark tests equal to the radial distance to each landmark on control tests. The results appear to fall between the two theoretical predictions in both versions of this experiment.

Figure 5 shows the median distances of searching on the two test types, along with the theoretical predictions of the independent averaging hypothesis. The median distance of search was calculated for each bee in each test type. Distance was measured from the north edge of the landmark on single-landmark tests and from the equivalent place on control tests (the line that is tangential to both landmarks on the target, north side of the landmarks). The distribution of searching within each bin was assumed to be flat. The theoretical predictions were made for each bee separately and then averaged across bees. In both experiments, the median distance of searching on the single-landmark tests fell between the two theoretical predictions.

Given that the single-landmark distributions were missing one bin, which was covered by the landmark, statistical tests were done on the median distances of search. The vector averaging hypothesis predicts equal search distances on the control and single-landmark tests. This was tested with a mixed model ANOVA with experiment as the between-subjects factor and test type as the within-subjects factor. Median distances of search differed significantly across experiments $[F(1,16)=$ $19.31]$ and across test types $[F(1,16)=33.06, p<.01]$, with no significant interaction. The significant test type main effect contradicts the vector averaging hypothesis, while the significant experiment main effect shows that the bees were sensitive to the training position of the target. To test the independent averaging hypothesis, the same ANOVA was applied to the single-landmark tests and the theoretical predictions, treated as two levels of "test type." The ANOVA yielded significant main effects of experiment $[F(1,16)=15.45]$ and test type $[F(1,16)=$ $126.90, p<.01]$, as well as a significant interaction $[F(1,16)=4.75]$. The main effect of test type contradicts the independent averaging model. The interaction suggests that the two experiments differed from the predictions of the independent averaging hypothesis by different amounts.

The statistical analysis thus confirms the impression that the results fell between the predictions of the two hypotheses. It is possible that an artifact was created because the bees could not search in one of the bins on single-landmark tests. To partially control for this, the ANOVAs were repeated with one bin in the control tests removed (the one that would have been covered by the landmark on single-landmark tests). Thus, the medians on control tests were recalculated and ANOVAs were redone. The same pattern of statistical results emerged. This does not, however, rule out the artifact completely, and Experiment 3 was in part an attempt to address this issue.

In sum, Experiment 2 allowed the measurement of the radial distance of search on single-landmark tests. The results were ambiguous in that they fell between the predictions of the vector averaging and independent averaging hypotheses. Interpretation of this intermediate pattern of results is addressed after all the results have been presented. 


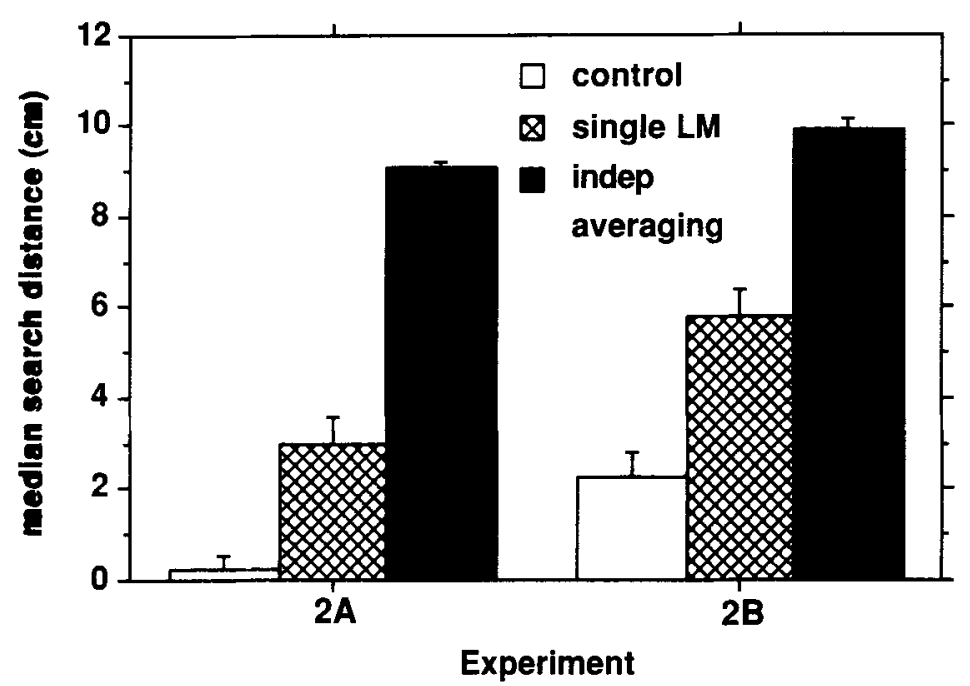

Figure 5. Median search distances in Experiments $2 A$ and 2B $(+S E M)$, with the theoretical predictions of the independent averaging hypothesis. The vector averaging hypothesis predicts the same search distances on control and single-landmark tests. LM, landmark.

\section{EXPERIMENT 3}

One objection to Experiment 2 is that on singlelandmark tests, the landmark was on the strip. This meant that the bees could not be searching in certain places on the strip during single-landmark tests, whereas they could search anywhere on the strip during control tests. Perhaps this artifactually made the search distributions different. Experiment 3 was performed to eliminate this potential artifact and provide another test of both hypotheses.

\section{Method}

Twenty bees participated, 10 bees in each of two conditions trained in two lots of 5 . The setups are shown in Figure 6. For the experimental group, two training setups were used, differing in the placement of the yellow strip (Figure 6A). The two blue-colored bottles (7-cm diameter, $15-\mathrm{cm}$ height) were $25 \mathrm{~cm}$ apart edge to edge. The yellow strip was now under one of the landmarks, oriented at $60^{\circ}$ clockwise or counterclockwise from the perpendicular line between the two landmarks. The front (northwest or northeast end) of the landmark was now on a grid line of the yellow strip. The target was $15 \mathrm{~cm}$ away from each landmark. Training took longer (30-60 trials) because the bees needed to learn two training setups.

Three kinds of tests were given. Two types of control tests used the setups of the two training arrays, but without reward. On a single-landmark test (Figure 6B), only one bottle was placed on the strip, which now ran along the short axis of the table, at the orientation exactly in between the orientations of the yellow strip on the two training setups. The front of the bottle (north edge) was on a grid line.

For the single-landmark control group, the training conditions were identical to those for the single-landmark test for the experimental group except that rewards were given on training trials at $15-\mathrm{cm}$ distance from the landmark. Bees were given 33-46 train- ing trials to roughly equate the duration of training between the two groups. This group, trained and tested with a single landmark, should search at about $15 \mathrm{~cm}$ from the landmark. Their behavior tested for any tendency to search closer than the training distance on single-landmark tests, irrespective of training history.

\section{Results and Discussion}

The search distributions of Experiment 3 are shown in Figure 7 . In this experiment, the radial distance to the landmark was measured for all tests. Thus, the independent averaging hypothesis now predicts the same distance for peaks of searching across tests. Trigonometric calculations show that the vector averaging hypothesis predicts a median search distance on single-landmark tests to be half the median search distance on control tests. The median distances of searching, calculated in the same way as in Experiment 2, are shown in Figure 8. The actual results on single-landmark tests once again fell between the two theoretical predictions. The theoretical prediction of the independent averaging hypothesis was the average of the two control tests, and the theoretical prediction of the vector averaging, according to vector geometry, was half that. Comparing the medians from the three empirical distributions with an ANOVA revealed that they differed significantly $[F(2,9)=4.79]$. An a priori contrast testing the independent averaging hypothesis is the single-landmark test against the average of the control tests. This contrast $[F(1,9)=8.99$, $p<.01]$ contradicted the predictions of the independent averaging hypothesis. A within-subjects comparison of the single-landmark tests against the predictions of the vector averaging hypothesis also showed a significant ef- 

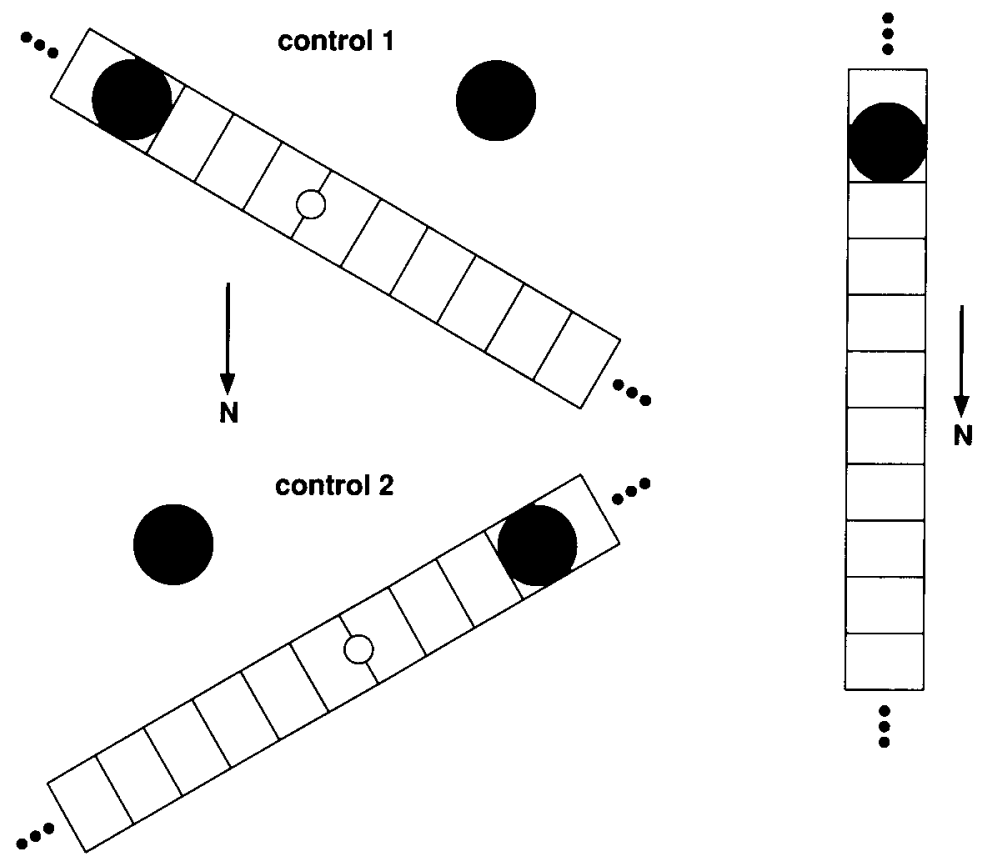

Figure 6. Setups used in Experiment 3. The filled circles represent light-blue cylindrical landmarks. The strip was light yellow, with pencil lines drawn $5 \mathrm{~cm}$ apart. Dots indicate that the strip continued the width of the table. The small open circle represents the target (dish of sugar water). Lab entrance is at the bottom of the figure (N). A: Training conditions and control tests. B: Single-landmark tests.

fect $[F(1,9)=92.31, p<.01]$, contradicting the predictions of the vector averaging hypothesis. Once again, neither averaging hypothesis was supported by the data.

The results for the single-landmark control group are also shown in Figures 7 and 8. Their search distribution looked like those of the control tests in the experimental group except that the levels of searching were higher overall (Figure 7). The median search distance for the single-landmark control group confirms this impression, the mean being in between the two control tests of the experimental group (Figure 8). On this measure, no overlap was found between the single-landmark control group and the single-landmark test for the experimental group. Clearly, the single-landmark test in and of itself did not lead bees to search at a shorter distance from the landmark than the training distance. Training with two landmarks in the experimental group was responsible for the different search distance found on single-landmark tests in that group.

The results of Experiment 3 thus replicate the pattern of Experiment 2, this time with a different training methodology. The search distance on single-landmark tests again came out somewhere between the predictions of vector averaging and independent averaging. At this point, several interpretations can be given. The first (Interpretation 1) is that the results really show vector averaging, but they have been distorted by a few centimeters; in other words, search distances reflect vector averaging plus some constant. A second, equally likely, possibility (Interpretation 2) is that the results show independent averaging with a few centimeters of distortion; in other words, search distances reflect independent averaging minus some constant. A variant of this interpretation, raised by a reviewer, is that independent averaging reigned, but that in addition, the honeybees had a tendency to approach the landmark on single-landmark tests. The landmark was after all in an unusual location, and one landmark was missing, so that exploration of the landmark might have been triggered. This tendency to approach the landmark distorted results by a few centimeters from the predictions of independent averaging. A third possibility (Interpretation 3 ) is that the results were truly intermediate, about halfway between the predictions of the two hypotheses. This could arise if the animals use both kinds of averaging mechanisms - an average of two modes of averaging. Because the difference between the predictions of the two hypotheses was similar in all three experiments, the three interpretations cannot be distinguished with the extant data. A fourth possibility (Interpretation 4) is that the data reflect multiple peaks on single-landmark tests that have merged together because the distances involved were small. This interpretation may be compatible with any of the underlying mechanisms postulated in Interpretations $1-3$. The nature of the peaks would pre- 


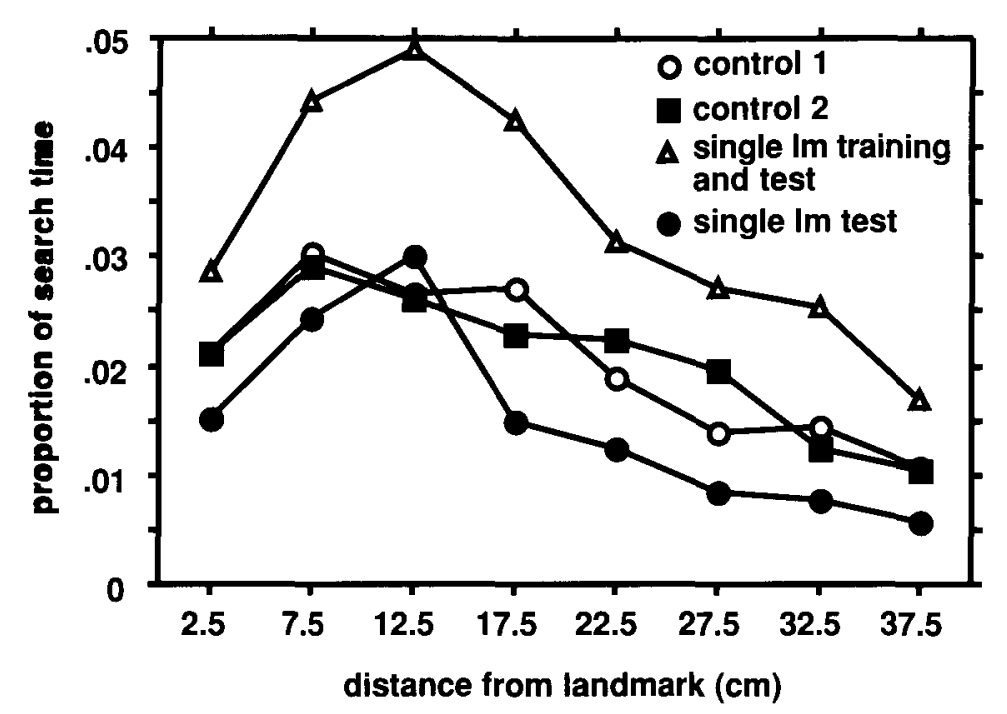

Figure 7. Search distributions in Experiment 3. The single-landmark control group was trained with the target $15 \mathrm{~cm}$ from a single landmark and tested only with the training setup. The experimental group was trained with the two training setups shown in Figure 6A. They were tested with the two training setups plus the singlelandmark test.

sumably reflect the kinds of underlying mechanisms used by the honeybees.

\section{EXPERIMENT 4}

Experiment 4 was an attempt to sort out the various interpretations of the data pattern obtained so far. The paradigm of Experiment 2 was repeated with much larger training distances: Landmarks were placed at the edges of the table. This increased the absolute difference in the predictions of the two hypotheses, hopefully making it easier to distinguish the interpretations. Now the four interpretations make different predictions for peaks on single-landmark tests. Interpretation 1 predicts a single peak a few centimeters too long from the predictions of the vector averaging hypothesis. Interpretation 2 predicts a single peak a few centimeters too short from the predictions of the independent averaging hypothesis. Interpre-

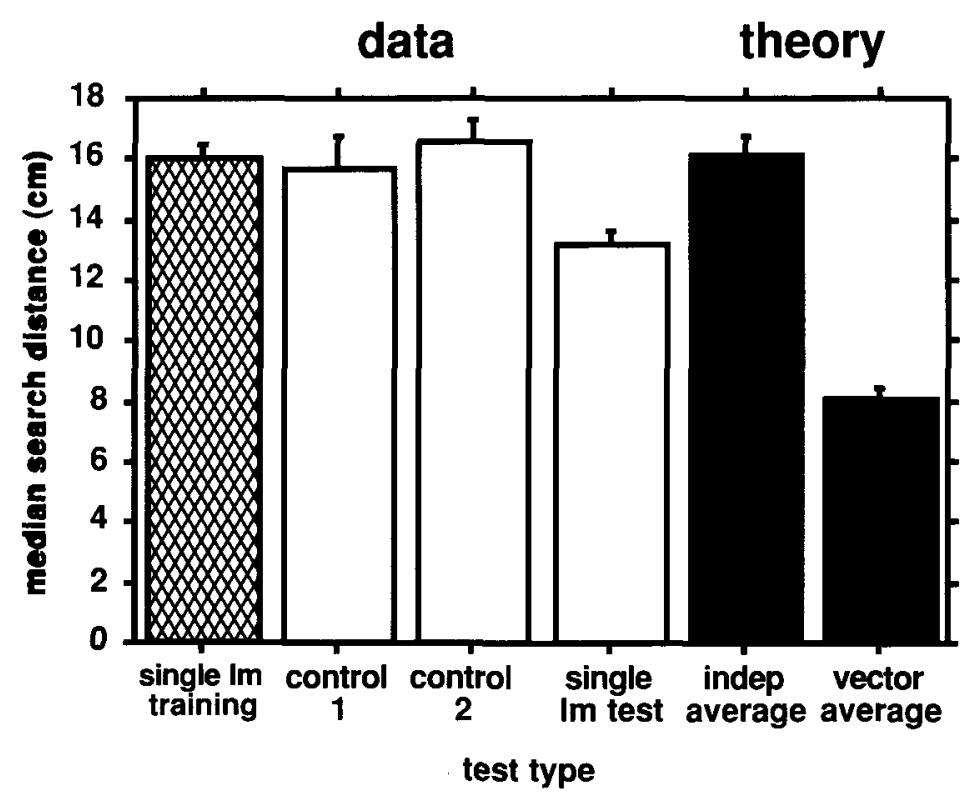

Figure 8. Median distance of search in Experiment $3(+S E M)$. The theoretical prediction of the independent averaging hypothesis was the average of the two control tests. The theoretical prediction of the vector averaging hypothesis, calculated by vector geometry, was half that. $L M$, landmark. 
tation 3 predicts a single peak somewhere intermediate between the predictions of the two hypotheses. Interpretation 4 predicts multiple peaks on single-landmark tests, with the location of the peaks dictating the nature of the interpretation.

\section{Method}

Ten bees participated, trained in two lots of 5 . The same table was used for this experiment. The yellow strip of $7-\mathrm{cm}$ width was placed along the length of the table, at the center. The strip was not moved during the experiment. Two cylindrical landmarks of the same color blue were used, measuring $12 \mathrm{~cm}$ in diameter and $25 \mathrm{~cm}$ in height. These were placed at the edges of the table. From the target, placed on the yellow strip on a grid line, the landmarks spanned $120^{\circ}$. Target-landmark distance was $32 \mathrm{~cm}$. The landmarks were west of the target (to the right of the target from the viewpoint of the entrance). The training array was moved along the yellow strip from trial to trial. Training followed the procedures of Experiment 2. Bees were given 50 training trials before tests began.

The same two kinds of tests as those used in Experiment 2 were used: control tests and single-landmark tests, four each per bee. On a single-landmark test, the west side of the bottle (the right side from the viewpoint of the entrance) lined up with a grid line. The vector averaging hypothesis predicts a search distance of about 13 $\mathrm{cm}$ (exact prediction depends on performance on control tests), whereas the independent averaging hypothesis predicts a search distance of about $32 \mathrm{~cm}$. The test procedure was varied slightly from that of Experiment 2. Before a bee arrived for a test, a red cardboard strip $50 \mathrm{~cm}$ long and $7 \mathrm{~cm}$ wide was placed on top of the yellow strip. On a single-landmark test, the near end of the strip was $3 \mathrm{~cm}$ from the edge of the landmark. On a control test, the strip was placed at the analogous location. It thus marked a distance of $50 \mathrm{~cm}$, starting at $3 \mathrm{~cm}$ in front of the east edge of the landmarks. This strip served as a template for a semiautomatic movement tracking software recently developed in our lab, and it was videotaped for a few seconds before a test. Then it was removed for the duration of the test. No bee ever saw the red strip. It was used purely for marking reference locations on video.

Data analysis relied on a movement tracking system that was developed in-house and based on the Image software of the National Institutes of Health (available at http://rsb.info.nih.gov/nih-image/ default.html). For each videotaped test, the system takes an average frame of the entire test. The average is then subtracted from each frame. Because the bee is the darkest object, what stands out after the subtraction process is usually the image of the bee. Filtering by object size allowed us to discard most of the points and lines that resulted from noise on the video record. Checking through the finished results allowed us to discard the rest of the wrongly identified objects (objects that were not the bee). Taking pixel measurements of the corners of the template (red strip videotaped before each test) allowed us to interpret the $x, y$ pixel measurements of the bee's location. The pixel measurements were translated to distance in centimeters from the east edge of the landmark (on single-landmark tests) or from the tangent to the east edges of landmarks on control tests. For data analysis, the data of concern were the frames in which the searching bee was on the yellow strip. For each such frame, the distance from the landmark was computed. The distance could be negative on control tests since the strip extended past the east tangent to the landmarks. On control tests, the range from -10 to $55 \mathrm{~cm}$ was included for analysis since this was the range in which data from all tests were available. The range included for analysis on single-landmark tests was 0 to $55 \mathrm{~cm}$.

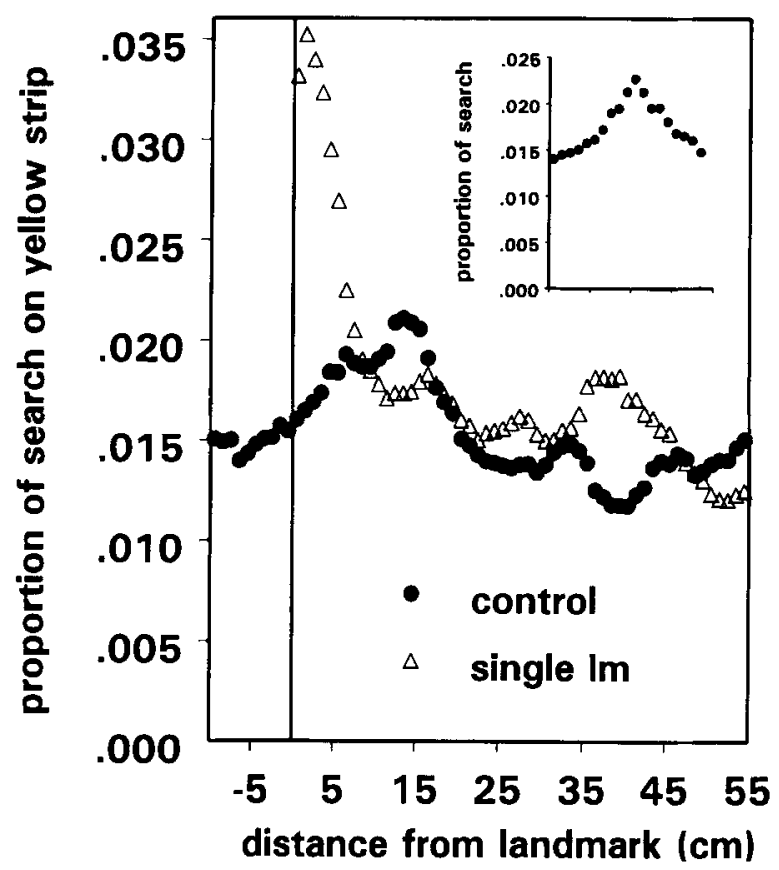

Figure 9. Search distributions in Experiment 4. Each point represents an average over a $5-\mathrm{cm}$ range. The dependent measure is the proportion of frames out of all frames in which the bee was over the yellow strip. The inset shows distribution on single-landmark tests when the modal bins on the right side for all bees were aligned. Data from 8 bees. LM, landmark.

\section{Results and Discussion}

The pooled search distributions (Figure 9) showed a single peak from the control tests, peaking at roughly the training distance of $13 \mathrm{~cm}$, and double peaks from the single-landmark tests. One peak, the highest one, was very close to the landmark (landmark edge at $0 \mathrm{~cm}$; vertical line in Figure 9). The right peak was found in each single bee's distribution. In the pooled distributions, this finding is muddied by the fact that different animals had peaks at different places. When the modal bins of the right peak were aligned for all the animals (inset, Figure 9), the right peak was clearly evident.

Each point in Figure 9 represents an average over a $5-\mathrm{cm}$ range, with neighboring points $1 \mathrm{~cm}$ apart (hence rolling 5 -cm averages). Edges of the ranges were suitably truncated (e.g., the point at $0.5 \mathrm{~cm}$ on single-landmark tests averaged from 0 to $3 \mathrm{~cm}$ ). Calculating the peak place of search for individual honeybees required using some measure other than the median. Because the peaks were not high, and the signal to noise ratio was low, the median was highly distorted by noise. Individual peak places were calculated as the middle of the largest peak region, as follows. For each distribution of each bee that went into Figure 9, a mean and a standard deviation were calculated on the $5-\mathrm{cm}$ rolling averages over the entire range 
of space. The locations with search durations $1 S D$ or more above the mean were marked. The widest range of marked locations with no more than two unmarked locations in between was taken to be the peak range (in fact, all unmarked gaps were only one bin wide or else more than four). The peak range included all locations that went into any bin of rolling averages. For example, if the marked range was from 12.5 to $18.5 \mathrm{~cm}$, the peak range ran from 10 to $20 \mathrm{~cm}$. The peak was the median of all the frames that fell in the peak range. The calculation of peaks from the control tests revealed 2 bees with aberrant peaks more than $10 \mathrm{~cm}$ from the training distance of $13 \mathrm{~cm}$ (one at 1.5 and one at $42.2 \mathrm{~cm}$ ). The results from these 2 bees were discarded, and Figure 9 shows results from the remaining 8 bees.

For the single-landmark distribution, a secondary peak was also calculated. Statistical justification for assuming two peaks is given below. For each bee separately, the minimum bin between the tallest two local maxima was found. The range from this local (and possibly global) minimum to the end of the range not containing the primary peak was taken. The process of peak calculation was repeated over this range. That is, means and standard deviations over this range were calculated, bins over $1 S D$ above the mean were marked, and so on. In this fashion, a left and a right peak were calculated for the single-landmark distributions. For 7 of 8 bees, the primary peak was the left peak, but for 1 bee, the primary peak was the right peak.

An ANOVA was conducted over the spatial range 0 to $55 \mathrm{~cm}$ of both distributions, using frame counts in rolling $5-\mathrm{cm}$ averages as a dependent measure (thus an absolute rather than a relative measure of search time). This ANOVA revealed that all the statistical effects were significant: location $[F(54,378)=6.92]$, test type $[F(1,7)=30.35]$, and the interaction $[F(54,378)=4.45]$. The test type main effect shows that the bees searched more on singlelandmark tests than on control tests. The interaction shows that this is attributable largely to the great amount of searching near the landmark on single-landmark tests. To analyze the nature of peaks more clearly, the singlelandmark distribution was examined alone. Over its entire range, a location main effect was found $[F(54,378)=$ $6.65]$. Over the range of $11 \mathrm{~cm}$ (location of first local minimum in the pooled distribution) to $55 \mathrm{~cm}$, a location main effect was also found $[F(43,301)=2.13]$. This justifies the search for a right peak. The range 11 to $25 \mathrm{~cm}$ (two local minima) was tested as well for a possible third peak. But in this range, no significant location effect was found $[F(13,91)=1.32]$. Thus statistical evidence can be found for two, but not three, peaks in the distribution for the single-landmark tests.

Figure 10 shows the peak places of searching, one for control tests and two for single-landmark tests, as well as the theoretical predictions of the independent averaging hypothesis. These theoretical predictions are calculated in the same way as in Experiment 2. The prediction of the vector averaging hypothesis is that the peak in the single-landmark distribution should equal that found in the control distribution. Inspection of Figure 10 shows that the left peak on single-landmark tests falls far shorter than the predictions of the vector averaging hypothesis, while the right peak matches the predictions of the independent averaging hypothesis. Formal statistics confirms these intuitions. An ANOVA comparing the right peak on single-landmark tests with the theoretical predictions of the independent averaging hypothesis revealed no significant differences $[F(1,7)=0.35]$, while

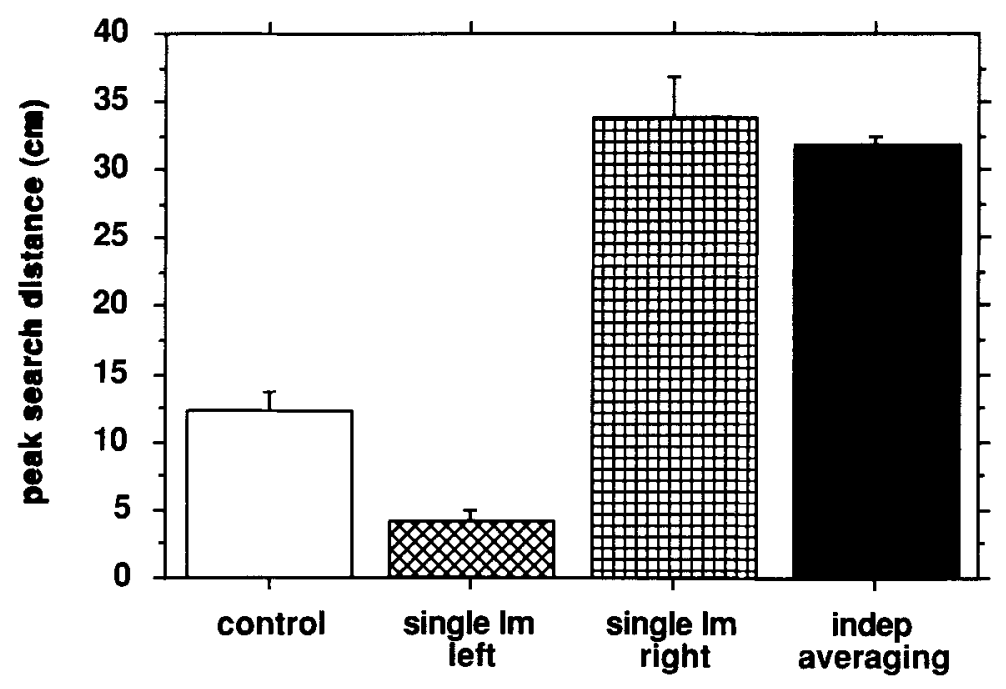

Figure 10. Peak search distances in Experiment 4 $(+S E M)$, with the theoretical predictions of the independent averaging hypothesis. Two peaks were calculated for singlelandmark tests. The vector averaging hypothesis predicts the same search distances on control and single-landmark tests. LM, landmark. 
an ANOVA comparing the control peaks with the left peak on single-landmark tests revealed significant differences $[F(1,7)=22.85, p<.01]$.

The two separate peaks on the single-landmark tests suggest that two different processes contribute to the peaks. The right peak can be interpreted as resulting from independent averaging of distances and directions: It is roughly at the same distance from the single landmark as the search peaks on control tests are from each of the landmarks in the training setup. The left peak is very close to the landmark, too close for the predictions of vector averaging, and probably reflects the approach to and exploration of a landmark in a new location. Such a pattern has been found in other studies (e.g., Cheng et al., 1987).

In sum, this experiment yielded evidence for independent averaging but not for vector averaging. The two processes of independent averaging and approaching a landmark in a new location can account for all the data in all the present experiments.

\section{GENERAL DISCUSSION}

Experiment 1 showed that honeybees averaged the dictates of two landmarks. They were trained with two landmarks, one on either side of the target. When tested with a single landmark, they searched more at an intermediate direction between the directions to the training landmarks than they did in control tests. Experiments $2 \mathrm{~A}$, $2 \mathrm{~B}$, and 3 also used two landmarks in training and tested bees with either one or two landmarks. The radial distance of search (distance from the landmark) on singlelandmark tests was measured and compared with the predictions of the vector averaging and independent averaging hypotheses. Results in all three cases came out in between the predictions of the two hypotheses. Experiment 4 used larger training distances and isolated two peaks on single-landmark tests. One peak corresponded with the predictions of the independent averaging hypothesis. The other peak was very close to the landmark and can best be interpreted as approach and exploration of the landmark.

These two processes of independent averaging and approach to the landmark can account for the results of Experiments 2 and 3 as well. The intermediary pattern of results suggests two processes at work, and the sensitivity of search peaks to training distances indicates that the bees were using the training landmarks on both singlelandmark tests and control tests. It is likely that the small distances used in Experiments 2 and 3 obscured any double peaks. It is possible that vector averaging also occurred but was somehow obscured by the process of approaching the landmark. Although such a possibility should be acknowledged, to date the data have not indicated clear evidence in its favor.

The evidence for independent averaging confirms an assumption of the models of Cartwright and Collett (1982,
1983). Comparatively, independent averaging of distances and directions has also been found in landmarkbased search in pigeons, a vertebrate species (Cheng, 1994). Perhaps this reflects convergent evolution dictated by the problem of locating places using nearby landmarks. Investigation of more species would help in settling this issue.

In measuring distances and directions to a stable landmark, it may be advantageous to compute the components separately. Computations on distance would not affect computations on direction, and vice versa. Separate computations mean that different specialized units, cognitive and neurophysiological, may be dedicated to each task.

We now have the following outline for how a honeybee navigates from its home to a foraging spot. It gets to the region of the target by following a vector or route (Wehner \& Menzel, 1990). It uses the sun compass and major landmarks to determine direction (Dyer \& Dickinson, 1996; Dyer \& Gould, 1983). Distance is measured chiefly by the visual flow of objects while the bee is flying (Esch \& Burns, 1995; Srinivasan, Zhang, \& Bidwell, 1997) or walking (Schöne, 1996; in ants, Ronacher \& Wehner, 1995). Once nearby landmarks are sighted, it beacons in on identified landmarks (Collett \& Rees, 1997). Landmarks are recognized by color (Cheng et al., 1986). At a certain point during its approach, the bee takes a sensorimotor trajectory that leads it toward the vicinity of the target (Collett \& Rees, 1997). In averaging trajectories, the bee uses vector averaging (Collett et al., 1996). If it sees the target, the bee beacons in on it (Collett \& Rees, 1997). If not, landmark matching is initiated. The bee flies facing one direction (Collett \& Baron, 1994) and attempts to put the landmarks at the correct positions on its eyes. Further, this paper provides clear evidence for Cartwright and Collett's $(1982,1983)$ claim that honeybees compute distances and directions to landmarks separately.

\section{REFERENCES}

AlYAN, S., \& JANDER, R. (1994). Short-range homing in the house mouse, Mus musculus: Stages in the learning of directions. Animal Behaviour, 48, 285-298.

BenNetT, A. D. T. (1993). Spatial memory in a food storing corvid. 1. Near tall landmarks are primarily used. Journal of Comparative Physiology A, 173, 193-207.

Biegler, R., \& Morris, R. G. M. (1993). Landmark stability is a prerequisite for spatial but not discrimination learning. Nature, 361, 631-633.

BIEGLER, R., \& MORRIS, R. G. M. (1996). Landmark stability: Further studies pointing to a role in spatial learning. Quarterly Journal of Experimental Psychology B, 49, 307-345.

Brodbeck, D. R. (1994). Memory for spatial and local cues: A comparison of a storing and a nonstoring species. Animal Learning \& Behavior, 22, 119-133.

Cartwright, B. A., \& Collett, T. S. (1982). How honeybees use landmarks to guide their return to a food source. Nature, 295,560 564.

Cartwright, B. A., \& Collett, T. S. (1983). Landmark learning in bees. Journal of Comparative Physiology A, 151, 521-543. 
CHENG, K. (1986). A purely geometric module in the rat's spatial representation. Cognition, 23, 149-178.

CHENG, K. (1988). Some psychophysics of the pigeon's use of landmarks. Journal of Comparative Physiology A, 162, 815-826.

Cheng, K. (1989). The vector sum model of pigeon landmark use. Journal of Experimental Psychology: Animal Behavior Processes, $15,366-375$.

CHENG, K. (1990). More psychophysics of the pigeon's use of landmarks. Journal of Comparative Physiology A, 166, 857-863.

Cheng, K. (1994). The determination of direction in landmark-based spatial search in pigeons: A further test of the vector sum model. $A n$ imal Learning \& Behavior, 22, 291-301.

CHENG, K. (1995). Landmark-based spatial memory in the pigeon. In D. L. Medin (Ed.), The psychology of learning and motivation (Vol. 33, pp. 1-21). San Diego: Academic Press.

Cheng, K., Collett, T. S., Pickhard, A., \& Wehner, R. (1987). The use of visual landmarks by honeybees: Bees weight landmarks according to their distance from the goal. Journal of Comparative Physiology A, 161, 469-475.

Cheng, K., Collett, T. S., \& Wehner, R. (1986). Honeybees learn the colour of landmarks. Journal of Comparative Physiology A, 159, 6973.

ChEng, K., \& Sherry, D. (1992). Landmark-based spatial memory in birds (Parus atricapillus and Columba livia): The use of edges and distances to represent spatial positions. Journal of Comparative Psychology, 106, 331-341.

Cheng, K., \& Spetch, M. L. (1995). Stimulus control in the use of landmarks by pigeons in a touch-screen task. Journal of the Experimental Analysis of Behavior, 63, 187-201.

COLLETT, T. S. (1992). Landmark learning and guidance in insects. Philosophical Transactions of the Royal Society of London: Series B, 337, 295-303.

Collett, T. S., \& BARon, J. (1994). Biological compasses and the coordinate frame of landmark memories in honeybees. Nature, $\mathbf{3 6 8}$ 137-140.

Collett, T. S., \& BARON, J. (1995). Learnt sensori-motor mappings in honeybees: Interpolation and its possible relevance to navigation. Journal of Comparative Physiology A, 177, 287-298.

Collett, T. S., Baron, J., \& Sellen, K. (1996). On the encoding of movement vectors by honeybees. Are distance and direction represented independently? Journal of Comparative Physiology A, 179, 395-406.

Collett, T. S., Cartwright, B. A., \& SMith, B. A. (1986). Landmark learning and visuo-spatial memories in gerbils. Journal of Comparative Physiology $A, 158,835-851$.

Collett, T. S., \& Kelber, A. (1988). The retrieval of visuo-spatial memories by honeybees. Journal of Comparative Physiology $A, \mathbf{1 6 3}$, 145-150.

Collett, T. S., \& ReES, J. A. (1997). View-based navigation in Hymenoptera: Multiple strategies of landmark guidance in approach to a feeder. Journal of Comparative Physiology A, 181, 47-58.

COLLETT, T. S., \& ZEIL, J. (1998). Places and landmarks: An arthropod perspective. In S. Healy (Ed.), Spatial representation in animals (pp. 18-53). Oxford: Oxford University Press.

DYER, F. C. (1991). Bees acquire route-based memories but not cognitive maps in a familiar landscape. Animal Behaviour, 41, 239-246.

Dyer, F. C., \& Dickinson, J. A. (1996). Sun-compass learning in insects: Representation in a simple mind. Current Directions in Psychological Science, 5, 67-72.

DYER, F. C., \& Gould, J. L. (1983). Honey bee navigation. American Scientist, 71, 587-597.

Esch, H. E., \& BurNS, J. E. (1995). Honeybees use optic flow to measure the distance of a food source. Naturwissenschaft, 82, 38-40.

Etienne, A. S., Berlie, J., Georgakopoulos, J., \& Maurer, R. (1998). Role of dead reckoning in navigation. In S. Healy (Ed.), Spatial representation in animals (pp. 54-68). Oxford: Oxford University Press.

Etienne, A. S., Joris-Lambert, S., Dahn-Hurni, C., \& Reverdin, B. (1995). Optimizing visual landmarks: Two and three dimensional minimal landscapes. Animal Behaviour, 49, 165-179.
Etienne, A. S., Joris-Lambert, S., Maurer, R., Reverdin, B., \& SitBON, S. (1995). Optimizing distal landmarks: Horizontal versus vertical structures and relation to background. Behavioural Brain Research, 68, 103-116.

Etienne, A. S., Teroni, E., Hurni, C., \& Portenier, V. (1990). The effect of a single light cue on homing behaviour of the golden hamster. Animal Behaviour, 39, 17-41.

Gallistel, C. R. (1990). The organization of learning. Cambridge, MA: MIT Press.

Gould, J. L. (1986). The locale map of honey bees: Do insects have cognitive maps? Science, 232, 861-863.

Gould, J. L. (1987). Landmark learning by honey bees. Animal Behaviour, 35, 26-34.

Gould-Beierle, K., \& Kamil, A. K. (1996). The use of local and global cues by Clark's nutcrackers (Nucifraga columbiana). Animal Behaviour, 52, 519-528.

HEALY, S. (ED.) (1998). Spatial representation in animals. Oxford: Oxford University Press.

Huber, B., Couvillon, P. A., \& Bitterman, M. E. (1994), Place and position learning in honeybees (Apis mellifera). Journal of Comparative Psychology, 108, 213-219.

KAMIL, A. C., \& JoNES, J. E. (1997). Clark's nutcrackers learn geometric relations between landmarks. Nature, 390, 276-279.

LEHRER, M., \& COLLETT, T. S. (1994). Approaching and departing bees learn different cues to the distance of a landmark. Journal of Comparative Physiology A, 175, 171-177.

Lehrer, M., Srinivasan, M. V., Zhang, S. W., \& Horridge, G. A. (1988). Motion cues provide the bee's visual world with a third dimension. Nature, 332, 356-357.

Margules, J., \& Gallistel, C. R. (1988). Heading in the rat: Determination by environmental shape. Animal Learning \& Behavior, 16, 404-410.

Mather, J. A. (1991). Navigation by spatial memory and use of visual landmarks in octopuses. Journal of Comparative Physiology A, 168, 491-497.

Menzel, R., Geiger, K., Jeorges, J., Müller, U., \& Chittka, L. (1998). Bees travel novel homeward routes by integrating separately acquired vector memories. Animal Behaviour, 55, 139-152.

Müller, M., \& Wehner, R. (1988). Path integration in desert ants, Cataglyphis fortis. Proceedings of the National Academy of Sciences, 85, 5287-5290.

RonaCher, B., \& WeHner, R. (1995). Desert ants Cataglyphis fortis use self-induced optic flow to measure distances travelled. Journal of Comparative Physiology A, 177, 21-27.

SCHÖNE, H. (1996). Optokinetic speed control and estimation of travel distance in walking honeybees. Journal of Comparative Physiology $A, 179,587-592$.

SPETCH, M. L. (1995). Overshadowing in landmark learning: Touchscreen studies with pigeons and humans. Journal of Experimental Psychology: Animal Behavior Processes, 21, 166-181.

Spetch, M. L., Cheng, K., \& MacDonald, S. E. (1996). Learning the configuration of a landmark array: I. Touch-screen studies with pigeons and humans. Journal of Comparative Psychology, 110, 5568

Spetch, M. L., Cheng, K., MacDonald, S. E., Linkenhoker, B. A., KeLLY, D. M., \& DoERKSON, S. R. (1997). Learning the configuration of a landmark array in pigeons and humans, II: Generality across search tasks. Journal of Comparative Psychology, 111, 14-24

SPETCH, M. L., ChenG, K., \& Mondloch, M. V. (1992). Landmark use by pigeons in a touch-screen spatial search task. Animal Learning \& Behavior, 20, 281-292.

Spetch, M. L., \& Edwards, C. A. (1988). Pigeons', Columba livia, use of global and local cues for spatial memory. Animal Behaviour, 36, 293-296.

SpetCh, M. L., \& Mondloch, M. V. (1993). Control of pigeons' spatial search by graphic landmarks in a touch-screen task. Journal of Experimental Psychology: Animal Behavior Processes, 19, 353372 .

Spetch, M. L., \& Wilkie, D. M. (1994). Pigeons' use of landmarks presented in digitized images. Learning \& Motivation, 25, 245-275. 
Srinivasan, M. V., Lehrer, M., Zhang, S. W., \& Horridge, G. A (1989). How honeybees measure their distance from objects of unknown size. Journal of Comparative Physiology A, 165, 605-613.

Srinivasan, M. V., ZhaNG, S. W., \& Bidwell, N. J. (1997). Visually mediated odometry in honeybees. Journal of Experimental Biology, 200, 2513-2522.

Suzuki, S., Augerinos, G., \& Black, A. (1980). Stimulus control of spatial behavior on the eight-arm maze in rats. Learning \& Motivation, 11, 1-18.

TinBergen, N. (1972). The animal in its world. Cambridge, MA: Harvard University Press.

Vallortigara, G., Zanforlin, M., \& Pasti, G. (1990). Geometric modules in animals' spatial representations: A test with chicks ( $\mathrm{Gal}$ lus gallus domesticus). Journal of Comparative Psychology, 104 248-254.

VANDER WALL, S. B. (1982). An experimental analysis of cache recovery in Clark's nutcracker. Animal Behaviour, 30, 84-94.

von FrISCH, K. (1953). The dancing bees (D. Ilse, Trans.). San Diego: Harcourt Brace Jovanovich.

Wehner, R., Bleuler, S., Nievergelt, C., \& Shah, D. (1990). Bees navigate by using vectors and routes rather than maps. Naturwissenschaften, 77, 479-482.

WeHNER, R., \& Menzel, R. (1990). Do insects have cognitive maps? Annual Review of Neurosciences, 13, 403-414.

WEHNER, R., MichEL, B., \& ANTONSEN, P. (1996). Visual navigation in insects: Coupling of egocentric and geocentric information. Journal of Experimental Biology, 199, 129-140.

WEHNER, R., \& RäBER, F. (1979). Visual spatial memories in desert ants, Cataglyphis bicolor (Hymenoptera: Formicidae), Experientia, 35, 1569-1571.

WeHNer, R., \& SRinivasan, M. V. (1981). Searching behaviour of desert ants, genus Cataglyphis (Formicidae, Hymenoptera), Journal of Comparative Physiology A, 142, 315-338.

WEHNER, R., \& WEHNER, S. (1990). Insect navigation: Use of maps or Ariadne's thread? Ethology, Ecology, \& Evolution, 2, 27-48.

\section{APPENDIX}

\section{Proof That Any Weighted Average of Two Vectors Lies on} the Line Segment Connecting the Endpoints

Vectors in 2-D space can be represented algebraically as a pair of $x, y$ coordinates. The arrow end of the vector lies at $(\mathbf{x}, \mathbf{y})$, while the other ("starting") end of the vector lies at $(\mathbf{0}, \mathbf{0})$. Take any two vectors, and without loss of generality, suppose that they have the same $y$ value. This can be accomplished by rotating the $x, y$ coordinate frame to the appropriate position. Thus, the vectors can be represented as $(\mathbf{x 1}, \mathbf{y})$ and $(\mathbf{x} 2, \mathbf{y})$. Furthermore, again without loss of generality, let $\mathbf{x} 2=$ $\mathbf{x} \mathbf{1}+\mathbf{x} \mathbf{0}$ for some positive $\mathbf{x} \mathbf{0}$. (One of $\mathbf{x} \mathbf{1}$ or $\mathbf{x} \mathbf{2}$ must be larger; the case $\mathbf{x 1}=\mathbf{x} 2$ holds trivially.) A weighted average of $(\mathbf{x} \mathbf{1}, \mathbf{y})$ and $(\mathbf{x} 1+\mathbf{x} \mathbf{0}, \mathbf{y})$ is

$$
\mathbf{a} *(\mathbf{x} 1, \mathbf{y})+(\mathbf{1 f}-\mathbf{a}) *(\mathbf{x} \mathbf{1}+\mathbf{x} \mathbf{0}, \mathbf{y}),
$$

where a is a scalar between 0 and 1 .

Multiplying the scalar in Equation Al through each term gives

$$
\begin{aligned}
& (\mathbf{a} * \mathbf{x} \mathbf{1}, \mathbf{a} * \mathbf{y})+(\mathbf{x} \mathbf{1}+\mathbf{x} \mathbf{0}, \mathbf{y})-(\mathbf{a} * \mathbf{x} \mathbf{1}+\mathbf{a} * \mathbf{x} \mathbf{0}, \mathbf{a} * \mathbf{y}) \\
= & (\mathbf{a} * \mathbf{x} \mathbf{1}+\mathbf{x} \mathbf{1}+\mathbf{x} \mathbf{0}-\mathbf{a} * \mathbf{x} \mathbf{1}-\mathbf{a} * \mathbf{x} \mathbf{0}, \mathbf{a} * \mathbf{y}+\mathbf{y}-\mathbf{a} * \mathbf{y}) \\
& (\text { adding } x \text { and } y \text { components }) \\
= & (\mathbf{x} \mathbf{1}+\mathbf{x} \mathbf{0}-\mathbf{a} * \mathbf{x} \mathbf{0}, \mathbf{y}) \text { (canceling } \mathbf{a} * \mathbf{y}, \mathbf{a} * \mathbf{x} \mathbf{1} \text { out }) .
\end{aligned}
$$

Thus, Equation A1 has the same $y$ value as $(\mathbf{x} 1, \mathbf{y})$ and $(\mathbf{x} 2, \mathbf{y})$ and hence lies on the line joining the endpoints of the two vectors. Furthermore, $\mathbf{x} \mathbf{1} \leq \mathbf{x} \mathbf{1}+\mathbf{x} \mathbf{0}-\mathbf{a} * \mathbf{x} \mathbf{0} \leq \mathbf{x} \mathbf{1}+\mathbf{x} \mathbf{0}$, since $\mathbf{a}$ is a scalar between 0 and 1 . This means that (A1) lies between $(\mathbf{x} 1, \mathbf{y})$ and $(\mathbf{x} 1+\mathbf{x 0}, \mathbf{y})$. Q.E.D. 